\title{
EDITORIAL
}

Chronic myelogenous leukemia

\section{Do persons with chronic myeloid leukaemia have normal or near normal survival?}

\author{
Tomas Radivoyevitch ${ }^{1}$ - Davis Weaver ${ }^{2}$ - Brian Hobbs ${ }^{1}$ - Jaroslaw P. Maciejewski ${ }^{1} \cdot$ Rudiger Hehlmann $^{3}$. \\ Qian Jiang ${ }^{4} \cdot$ Andreas Hochhaus $^{5} \cdot$ Robert Peter Gale $^{6}$
}

Received: 13 October 2019 / Revised: 14 November 2019 / Accepted: 12 December 2019 / Published online: 20 December 2019

๑) Springer Nature Limited 2019

Recent clinical trial and population-based registry reports claimed survival of persons with chronic phase chronic myeloid leukaemia (CML) receiving tyrosine kinaseinhibitor (TKI) therapy, especially those achieving a complete cytogenetic response (CCyR; no detectable $\mathrm{Ph}^{1}$-chromosome) and/or a sustained deep molecular responses (DMR; $>4$ or $4.5 \log$ reduction in $B C R A B L 1$ transcripts), is similar to that of age- and sex-matched controls [1-4]. Is this so? Although there is a high response rate to TKItherapy, some responders relapse and a few develop blast transformation. Others become intolerant of and/or noncompliant with TKI-therapy whilst still others discontinue TKI-therapy because of fiscal considerations or adverse events [5]; 2nd and 3rd generation TKIs used in persons responding inadequately to $1 \mathrm{st}$ generation TKI-therapy associate with some adverse events which are lifethreating [6]. TKI-therapy can be successfully stopped in only about $20 \%$ of persons with CML (reviewed in $[7,8]$ ). Lastly, it is rather unlikely TKI-therapy eliminates CML stem cells in anyone (reviewed in [9]).

To determine if the most people with CML really have a normal life expectancy we used our software SEERaBomb [10] to interrogate the US Surveillance and Epidemiology End Results (SEER) dataset. To observed deaths (O) we

\footnotetext{
Robert Peter Gale

robertpetergale@alumni.ucla.edu

Cleveland Clinic Foundation, Cleveland, OH, USA

2 Case Western Reserve University, Cleveland, OH, USA

3 Universität Heidelberg and ELN-Foundation, Weinheim, Germany

4 Peking University Peoples Hospital, Peking University Institute of Hematology, Beijing, China

5 Abteilung Hämatologie-Onkologie, Universitätsklinikum Jena, Jena, Germany

6 Centre for Haematology, Department of Immunology and Inflammation, Imperial College, London, UK
}

fitted expected numbers $m$ equal to a modelled rate multiplied by deaths expected (E) if subject follow-up time intervals were randomly selected from the US population for the same age, sex and calendar year. Poisson regression yielded an excellent fit to relative risks (RR) of death $(\mathrm{O} / \mathrm{E}$ vs. $\mathrm{m} / \mathrm{E}$ in Fig. 1a). Relative to survival plots of the same data (Fig. 1b), advantages of RR include a standard reference line $(\mathrm{RR}=1)$ indicating how close we are to cure. Fitting $m=\left(1+k_{1} e^{c_{1}-k_{1} t}+k_{2} e^{c_{2}-k_{2} t}+c_{3}\right) E$ to $\mathrm{O}$, in the limit of large times $t$ since diagnosis, $c_{3}$ indicates persons with CML in the US have a 2.38 -fold (95\% confidence interval; $1.70,3.07 ; P<0.0001)$ higher risk of death than controls. Although the SEER dataset includes a small proportion of subjects with acute phase CML and with $\mathrm{Ph}^{1}$ chromosome-negative CML, this does not explain our finding of worse survival of persons with CML. Nor do differences in healthcare systems as life expectancy in the countries studied are similar.

Of possible explanations for the discordance between our conclusion and those of clinical trials and population-based registries, the most likely is access to, compliance with and monitoring of TKI-therapy. A real world US study of people with CML reported infrequent molecular monitoring of response to TKI-therapy [11]. We found a similar deficiency in molecular monitoring in China [12]. There also may be other explanations such as lack or insufficient access to healthcare. In a US population-based study, persons with $>85 \%$ access to TKIs via Medicare had comparable survival to age- and sex-matched controls [4], suggesting our finding of decreased survival comes from those on Medicare with $<85 \%$ access to TKI and/or other, likely under-insured, populations. In a study in China males, unmarried persons, persons with a low education level and those with rural residence had worse responses to TKI-therapy and worse survival compared with controls [13]. At a recent global CML meeting in Bordeaux in September, 2019, researchers from several other countries including Argentina and Columbia reported worse survival in persons with CML 


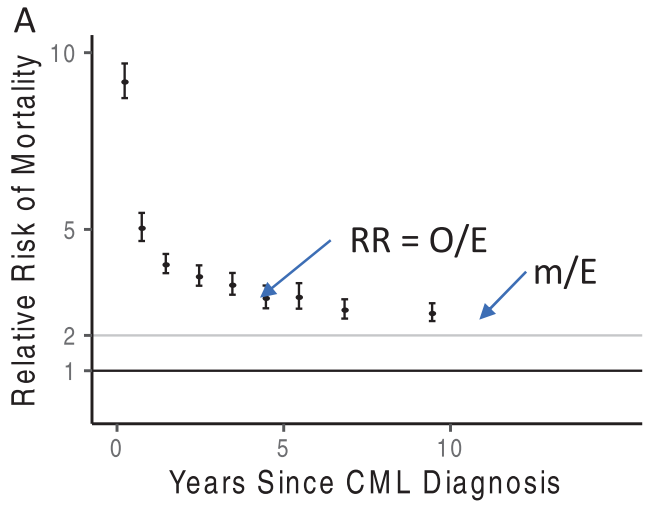

Fig. 1 SEER CML mortality. a The relative risk of CML mortality levels off at $\sim 2$, not $\sim 1$. b Observed vs. simulated/expected Kaplan-Meier survival plots using the same data as in A. CML cases diagnosed between 1/1/2002 and 12/31/2016 were used in this

compared with controls as well as correlations between type of heath care insurance and survival.

The disparity in survival of persons we detected in the unselected US SEER population and, for example, a Sweden population-based registry occurs despite the US spending about twice as much per capita on healthcare (2016 \$9,892 in International USD purchasing power parity [PPP]) compared with Sweden (2016 \$5488 International USD PPP) [14]. This disparity is also reflected in similar 2015 estimated average life expectancies despite higher US spending: US 79.3 years vs. Sweden, 82.4 years [15]. A final consideration is persons with CML receiving TKItherapy see physicians frequently, typically 3-4 times annually. Consequently, unrelated health problems may be diagnosed and treated earlier than in normals resulting in a compensatory increase in survival. However, most data suggest no survival benefit in the general population for frequent medical examinations [16]. Whether this is so for persons with CML on TKI-therapy is unknown.

Our message is caution is needed extrapolating results of therapy-interventions in subjects in clinical trials and population-based registries to people in other countries and with other healthcare systems. There is a twofold increased risk of death in persons in the US with CML and probably in many if not most other countries. Consider that only 5\% of persons with CML live in Europe. This is not only a problem in underdeveloped and developing countries. Many Americans (another 5\% of the global population) have inadequate health insurance coverage. Although we have effective therapies of CML getting them efficiently to people with CML globally remains challenging. More work is needed.

Acknowledgements TR acknowledges support from the Cleveland Clinic Foundation. BH acknowledges support from Amgen, SimulStat and Presagia. AH acknowledges research support from Novartis,

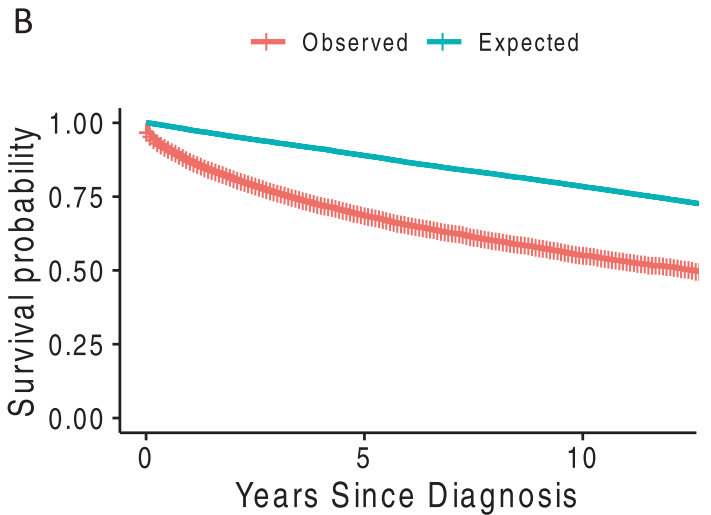

analysis. For additional details see the R script that generated these plots, france2019.R, in the papers/misc folder of SEERaBomb available from GitHub [https://github.com/radivot/SEERaBomb/tree/ma ster/SEERaBomb/inst/docs/papers/misc].

Bristol-Myers Squib, Pfizer and Incyte Corps. QJ acknowledges support from the National Natural Science Foundation of China (No. 81770161). RPG acknowledges support from the National Institute of Health Research (NIHR) Biomedical Research Centre funding scheme.

\section{Compliance with ethical standards}

Conflict of interest RPG is a part-time employee of Celgene Corp.

Publisher's note Springer Nature remains neutral with regard to jurisdictional claims in published maps and institutional affiliations.

\section{References}

1. Bower H, Björkholm M, Dickman PW, Höglund M, Lambert PC, Andersson TM. Life expectancy of patients with chronic myeloid leukemia approaches the life expectancy of the general population. J Clin Oncol. 2016;34:2851-7.

2. Hehlmann R, Lauseker M, Saussele S, Pfirrmann M, Krause S, Kolb HJ, et al. Assessment of imatinib as first-line treatment of chronic myeloid leukemia: 10-year survival results of the randomized CML study IV and impact of non-CML determinants. Leukemia. 2017;31:2398-406.

3. Gambacorti-Passerini C, Antolini L, Mahon, Guilhot F, Deininger M, Fava C, Nagler A, et al. Multicenter independent assessment of outcomes in chronic myeloid leukemia patients treated with imatinib. J Natl Cancer Inst. 2011;103:553-61.

4. Kenzik KM, Bhatia R, Williams GR, Bhatia S. Medicare and patient spending among beneficiaries diagnosed with chronic myelogenous leukemia. Cancer. 2019;125:2570-8.

5. Marin D, Bazeos A, Mahon FX, Eliasson L, Milojkovic D, Bua $\mathrm{M}$, et al. Adherence is the critical factor for achieving molecular responses in patients with chronic myeloid leukemia who achieve complete cytogenetic responses on imatinib. J Clin Oncol. 2010;28:2381-8.

6. Efficace F, Stagno F, Iurlo A, Breccia M, Cottone F, Bonifacio M, et al. Health-related quality of life of newly diagnosed chronic myeloid leukemia patients treated with first-line dasatinib versus imatinib therapy. Leukemia. 2019; https://doi.org/10.1038/ s41375-019-0563-0.

7. Saussele S, Richter J, Guilhot J, Gruber FX, Hjorth-Hansen H, Almeida A, et al. Discontinuation of tyrosine kinase inhibitor 
therapy in chronic myeloid leukaemia (EURO-SKI): a prespecified interim analysis of a prospective, multicentre, nonrandomised trial. Lancet Oncol. 2018;19:747-57.

8. Saglio G, Gale RP. Prospects for achieving therapy-free remission in chronic myeloid leukaemia. Br. J Haematol. 2020. In press.

9. Houshmand M, Simonetti G, Circosta P, Gaidano V, Cinetti A, Martinelli G, et al. Chronic myeloid leukemia stem cells. Leukemia. 2019;33:1543-56.

10. Radivoyevitch T, Sachs RK, Gale RP, Molenaar RJ, Brenner DJ, Hill BT, et al. Defining AML and MDS second cancer risk dynamics after diagnoses of first cancers treated or not with radiation. Leukemia. 2016;30:285-94.

11. Goldberg SL. Monitoring chronic myeloid leukemia in the real world: gaps and opportunities. Clin Lymphoma Myeloma Leuk. 2015;15:711-4.
12. Jiang Q, Gale RP. Molecular monitoring of tyrosine kinase inhibitor therapy of chronic myeloid leukemia in China. J Cancer Res Clin Oncol. 2016;142:1549-55.

13. Jiang Q, Yu L, Dou X. Impact of socio-demographics on disease phase and risk, drug choice, and treatment outcomes in adults with newly-diagnosed chronic myeloid leukemia. Presented at the 21st Annual John Goldman Conference on Chronic Myeloid Leukaemia: Biology and Therapy. Bordeaux, France.

14. https://stats.oecd.org/Index.aspx ?DataSetCode $=$ SHA. Accessed 19 Sep 2019.

15. https://www.who.int/en/news-room/detail/19-05-2016-lifeexpectancy-increased-by-5-years-since-2000-but-health-inequa lities-persist. Accessed 19 Sep 2019.

16. Mehrotra A, Prochazka A. Improving value in health care-against the annual physical. N. Engl J Med. 2015;373:1485-7. 